\title{
Biodegradable HEMA-based hydrogels with enhanced mechanical properties
}

\author{
Mohamadreza Nassajian Moghadam, Dominique P. Pioletti \\ Laboratory of Biomechanical Orthopedics, Institute of Bioengineering, École Polytechnique Fédérale de Lausanne (EPFL)
}

Received 20 January 2015; revised 30 April 2015; accepted 22 May 2015

Published online 9 June 2015 in Wiley Online Library (wileyonlinelibrary.com). DOI: 10.1002/jbm.b.33469

\begin{abstract}
Hydrogels are widely used in the biomedical field. Their main purposes are either to deliver biological active agents or to temporarily fill a defect until they degrade and are followed by new host tissue formation. However, for this latter application, biodegradable hydrogels are usually not capable to sustain any significant load. The development of biodegradable hydrogels presenting load-bearing capabilities would open new possibilities to utilize this class of material in the biomedical field. In this work, an original formulation of biodegradable photo-crosslinked hydrogels based on hydroxyethyl methacrylate (HEMA) is presented. The hydrogels consist of short-length poly(2-hydroxyethyl methacrylate) (PHEMA) chains in a star shape structure, obtained by introducing a tetra-functional chain transfer agent in the
\end{abstract}

backbone of the hydrogels. They are cross-linked with a biodegradable N,O-dimethacryloyl hydroxylamine (DMHA) molecule sensitive to hydrolytic cleavage. We characterized the degradation properties of these hydrogels submitted to mechanical loadings. We showed that the developed hydrogels undergo long-term degradation and specially meet the two essential requirements of a biodegradable hydrogel suitable for load bearing applications: enhanced mechanical properties and low molecular weight degradation products. (c) 2015 Wiley Periodicals, Inc. J Biomed Mater Res Part B: Appl Biomater, 104B: 1161-1169, 2016.

Key Words: HEMA-based hydrogels, biodegradable hydrogels, mechanical tests

How to cite this article: Nassajian Moghadam M, Pioletti DP. 2016. Biodegradable HEMA-based hydrogels with enhanced mechanical properties. J Biomed Mater Res Part B 2016:104B:1161-1169.

\section{INTRODUCTION}

Hydrogels are widely used in biomedical applications either as vehicles to deliver a biological agent or as scaffolds to fill a defect. In particular, the biodegradable version of hydrogels is of great interest since upon their degradation, formation of neo-tissues can be obtained in the defect. ${ }^{1-4}$ However, due to the weak mechanical properties of biodegradable hydrogels, their application is very limited in tissues functioning under load situations. ${ }^{5-8}$ Therefore, there is a clear need for developing biodegradable hydrogels with an enhanced load-bearing capability.

Biodegradable hydrogels may be produced based on the cleavage of hydrolytically or enzymatically labile bonds ${ }^{5-8}$ or dissolution of physical cross-links formed via hydrophobic, electrostatic, or hydrogen-bonding forces. ${ }^{9,10}$ Due to these weak cross-linking mechanisms, most of the developed biodegradable hydrogels exhibit poor mechanical properties. ${ }^{11,12}$ In contrast, synthetic chemically cross-linked hydrogels with strong covalent bonds present an enhanced mechanical strength. ${ }^{11,13,14}$ However, the strong covalent bonds limit the degradation of these hydrogels. ${ }^{11,14}$

In order to develop a hydrogel with simultaneously strong mechanical properties and degradation capabilities, we focused our attention on methacrylate polymers such as poly(2-hydroxyethyl methacrylate) (PHEMA). Hydrogels made of these polymers were shown to be biocompatible, to have tunable mechanical properties, and they can be fabricated in different architectures. ${ }^{15-17}$ We recently showed that HEMAbased hydrogels present very high load tolerance and especially resistance to crack propagation when we increased their dissipation properties. ${ }^{18}$ However, because of its high biostability, ${ }^{16}$ PHEMA has not been successfully used as a biodegradable hydrogel yet, despite several studies have focused on this aspect. ${ }^{6,17,19-22}$ Although some of the proposed structures presented degradation capabilities to some extent, the resulting hydrogels had two major deficiencies. First, as expected they showed very poor mechanical properties due to the structure of the cross-linker used ${ }^{11,22}$ or to the mechanism of cross-linking. ${ }^{23,24}$ Second, their degradation products consisted of very long PHEMA chains. ${ }^{6}$ Indeed, the structure of HEMA-based hydrogels is composed of long PHEMA chains interconnected with cross-linker molecules. It has been reported that under physiological conditions, PHEMA can be hydrolyzed to poly(methyl methacrylate). ${ }^{25}$ However, if the degradation occurs just via dissolution of the cross-linkers, the resulting degradation product consists of very long chains of coiled PHEMA fragments, which cannot be cleared out of the body. $6,26,27$ 
To overcome these two drawbacks, we developed an original formulation of biodegradable HEMA-based hydrogels, which present enhanced mechanical properties and degradation products with low molecular weight. These hydrogels were developed based on HEMA cross-linked with a short hydrolysable molecule N,O-dimethacryloyl hydroxylamine (DMHA) and employing a tetra-functional chain transfer agent in the backbone of these hydrogels. DMHA provides high initial mechanical properties and the tetra-functional molecule breaks down the PHEMA long backbone chains to small star-like molecules.

\section{MATERIALS AND METHODS}

\section{Materials}

All materials were purchased from Aldrich (Buchs, Switzerland) and were stored at $4^{\circ} \mathrm{C}$ until use unless otherwise specified. Hydroxylamine hydrochloride, methacryloyl chloride $\left(-20^{\circ} \mathrm{C}\right)$, pyridine, chloroform, and hydrochloric acid were all used for synthesizing N,O-dimethacryloyl hydroxylamine. 2-2hydroxyethyl methacrylate (HEMA, 97\%) was purified using basic aluminum oxide column chromatography to remove inhibitors. 2,2-dimethoxy-2-phenylacetophenone (DPAP) (Irgacure-651, 99\%) was used as photo initiator and prepared as an ethanolic solution of DPAP $(57 \mathrm{mg} / \mathrm{mL}$ solution, each $\mathrm{ml}=0.2 \mathrm{mM}$ ). Pentaerythritol tetrakis(3-mercaptopropionate) was used as received without further purification.

Cell culture media contained $10 \mathrm{~mL}$ Dulbecco's Modified Eagle Medium (DMEM) with $25 \mathrm{~m} M$ dextrose and $1 \mathrm{mM}$ sodium pyruvate (Life Technologies Ltd, Paisley, UK), $5.97 \mathrm{~m} M$ L-Glutamine (Life Technologies Ltd), 10\% fetal bovine serum (Sigma, St. Louis, MO). Giemsa's azur eosin methylene blue solution for microscopy (Merck, Darmstadt, Germany) and CellTiter (G3580 Promega, Fitchburg, WI) were used for the cell study.

\section{DMHA synthesis}

N,O-Dimethacryloyl hydroxylamine (DMHA) was synthesized following a slightly modified protocol initially proposed by South et al. $^{28}$ Ten grams of hydroxylamine hydrochloride ( $0.145 \mathrm{~mol})$ was added in a $500 \mathrm{~mL}$ round-bottom flask and put under $\mathrm{N}_{2}$ gas for $\sim 30 \mathrm{~min}$ to remove moisture. Totally, $50 \mathrm{~mL}$ pyridine was added by syringe and stirred with the hydroxylamine hydrochloride until complete dissolution. The solution was maintained under $\mathrm{N}_{2}$ and in an ice bath to keep the temperature of the reaction mixture below $20^{\circ} \mathrm{C}$. Methacryloyl chloride, $29 \mathrm{~mL}$ (0.3 mol), was added dropwise. We reduced the system's exposure to light as much as possible by covering the flask with an aluminum foil. The reaction was then stirred for an additional $5 \mathrm{~h}$. After complete conversion, $100 \mathrm{~mL}$ of chloroform was added, and the solution became a transparent yellow-brown liquid. Afterward, $100 \mathrm{~mL}$ of hydrochloric acid (1.5 molar) was added dropwise, while the solution was maintained in the ice bath and under aluminum foil. The mixture turned turbid. The solution was poured into a separatory funnel and was washed with $100 \mathrm{~mL}$ of deionized water until the aqueous layer became clear (four washes). The organic layer was then dried over magnesium sulfate and filtered. We removed chloroform by rotary evaporation in a
TABLE I. Hydrogel Composition

\begin{tabular}{lcccc}
\hline $\begin{array}{l}\text { Sample } \\
\text { Name }\end{array}$ & $\begin{array}{c}\text { HEMA } \\
(\% \mathrm{~mol})\end{array}$ & $\begin{array}{c}\text { DMHA } \\
(\% \mathrm{~mol})\end{array}$ & $\begin{array}{c}\text { Tetrakis } \\
(\% \mathrm{~mol})\end{array}$ & $\begin{array}{c}\text { Water } \\
(\% \text { Total } \\
\text { Volume })\end{array}$ \\
\hline S1 & 100 & 10 & 0 & 40 \\
S2 & 100 & 10 & 0.25 & 40 \\
S3 & 100 & 1 & 0 & 65 \\
S4 & 100 & 1 & 0.25 & 65 \\
\hline
\end{tabular}

dark $200 \mathrm{~mL}$ round bottom flask and then, the yellow oily product was dried overnight on a Schlenk line. The product was a yellow highly viscous liquid. Experimental yield was approximately 30\%. The chemical composition of DMHA was verified with ${ }^{1} \mathrm{H}$ NMR using a Varian Unity $300 \mathrm{MHz}$ NMR spectrometer (Fitchburg, MI). For ${ }^{1} \mathrm{H}$ NMR test, sample (10 $\mu \mathrm{L}$ ) was dissolved in DMSO- $\mathrm{d}_{6}$. The following chemical shifts

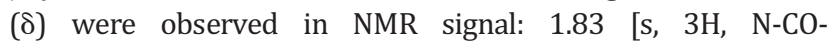
$\left.\mathrm{C}\left(\mathrm{CH}_{3}\right)=\mathrm{CH}_{2}\right], 1.97\left[\mathrm{~s}, 3 \mathrm{H},-\mathrm{CO}-\mathrm{C}\left(\mathrm{CH}_{3}\right)=\mathrm{CH}_{2}\right], 5.48,5.73[\mathrm{~s}$, $\left.2 \mathrm{H},-\mathrm{N}-\mathrm{CO}-\mathrm{C}\left(\mathrm{CH}_{3}\right)=\mathrm{CH}_{2}\right], 5.85,6.28[\mathrm{~s}, 2 \mathrm{H},-\mathrm{O}-\mathrm{CO}-$ $\left.\mathrm{C}\left(\mathrm{CH}_{3}\right)=\mathrm{CH}_{2}\right]$

\section{Hydrogels synthesis}

PHEMA hydrogels were prepared with $\mathrm{N}, \mathrm{O}$-dimethacryloyl hydroxylamine (DMHA) as cross-linker $(1 \%$ and $10 \%$ of mol) and water ( $65 \%$ and $40 \%$ of total mixture volume). In another group of hydrogels we added a four functional group molecule, pentaerythritol tetrakis(3-mercaptopropionate) $(0.25 \%$ of mol). The term "Tetrakis" will be used for this material in the following sections. Material compositions for all four groups of samples are summarized in Table I (S1, S2, S3, and S4). The mixture containing the photo initiator DPAP $(0.1 \%$ of mol) was stirred and sonicated for $1 \mathrm{~min}$. It was then transferred to cylindrical wells (6 $\mathrm{mm}$ diameter and $3 \mathrm{~mm}$ depth), placed under UV lamp (365 nm, $8 \mathrm{watt}$ ) (Upland, CA) positioned $10 \mathrm{~cm}$ from the samples and irradiated for $15 \mathrm{~min}$. The system was maintained below $25^{\circ} \mathrm{C}$ during polymerization by air circulation. Hydrogels were then carefully removed from the wells, washed to remove unreacted materials, and immersed in water for 1 week. Figure 1 shows the expected structure of the hydrogels with and without Tetrakis. In the presence of Tetrakis, hydrogels present a star structure in which four short PHEMA chains are linked by ester bonds to a tetrafunctional Tetrakis molecule. The branches of HEMA in the ideal case when monomer conversion reaches $100 \%$ contain an average number of units equal to $1 /$ (number of $\mathrm{SH}$ groups $\times$ Tetrakis to HEMA molar ratio). The number of $\mathrm{SH}$ groups on each Tetrakis is four. ${ }^{25}$ For the Tetrakis to HEMA, molar ratio equals to $0.25 \%$, the average number of units will be equal 100 HEMA molecules. The PHEMA branches are connected together with DMHA cross-linkers. Without Tetrakis hydrogels contain long chains of HEMA molecules coiled together and cross-linked with DMHA.

\section{Swelling and mechanical evaluation of hydrogels}

We studied the swelling behavior of hydrogels by measuring the equilibrium water content at swollen state after one 


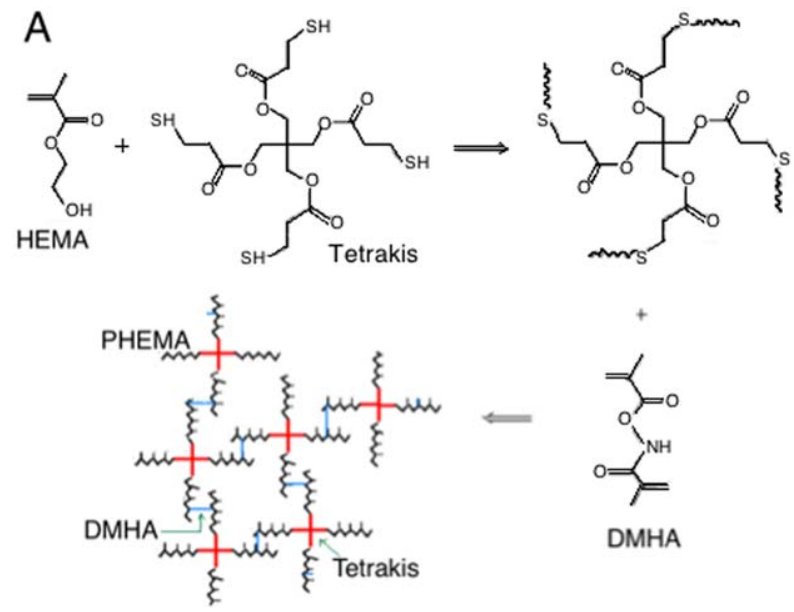

B

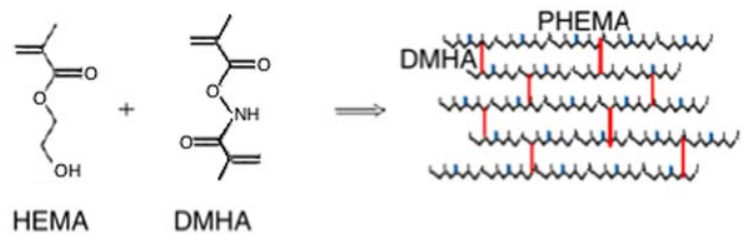

FIGURE 1. Structure of the hydroxyethyl methacrylate (HEMA) hydrogels. A: HEMA cross-linked with $\mathrm{N}, \mathrm{O}$-dimethacryloyl hydroxylamine (DMHA) in presence of Tetrakis (HEMA-DMHA-Tetrakis hydrogels). In the presence of Tetrakis, hydrogels present a star structure in which four short PHEMA chains are linked to a Tetrakis molecule. The PHEMA branches are connected together with DMHA cross-linkers. B: HEMA cross-linked with $\mathrm{N}, \mathrm{O}$-dimethacryloyl hydroxylamine (DMHA) in the absence of Tetrakis (HEMA-DMHA hydrogels). Without Tetrakis, hydrogels contain long chains of HEMA molecules cross-linked with DMHA.

week of swelling in PBS. We prepared six samples for each of the four hydrogel groups. The equilibrium water content was measured as the ratio of the swelling weight of the hydrogels minus their dried weight, over their dried weight. To dry hydrogels, each sample was first flash frozen in liquid nitrogen and then dried under vacuum (Dynavac FD2, Boronia, Australia) over 3 days. We also measured the initial water content as the ratio of the hydrogels' weight immediately after polymerization minus the dried weight, over the dried weight.

The elastic and viscous properties of the swollen samples were characterized by measuring the elastic modulus and the damping ratio of the hydrogels. For the elastic modulus, a single compressive load up to $10 \%$ deformation at the rate of $1 \%$ per second was applied with an Instron E3000 linear mechanical testing machine (Instron, Norwood, MA). In this range of deformation, each hydrogel had a linear stress-strain relationship. The elastic modulus was calculated from the slope of the stress-strain curve of each sample. For quantifying the damping ratio, cyclic compression test at $1 \mathrm{~Hz}$ and $15 \%$ deformation amplitude was applied on samples for $60 \mathrm{~s}$ with the Instron machine. The damping ratio was calculated as the ratio of the dissipated work (the area of the hysteresis curve in the forcedisplacement graph) over the total input work given to the material (the area under the compression curve in the force-displacement graph) during one cyclic deformation. ${ }^{29}$ We considered the mean value of damping ratio for the last 10 cycles.

\section{Accelerated degradation study}

It has been shown that basic environment expedites the hydrolysis of DMHA. ${ }^{30}$ In order to compare the degradation capacity of the produced hydrogels and evaluate the effect of different parameters like the cross-linker ratio, water content, and the presence of the Tetrakis molecules, the mass loss of thin films of hydrogels was measured during 30 days, when they were incubated in highly basic environment. The hydrogel thin films were prepared by cutting the hydrogel samples from each group of S1, S2, S3 and S4 with a Vibratome machine (Leica VT1200S, Muttenz, Switzerland) in $300 \mu \mathrm{m}$ films $(4 \mathrm{~mm} \times 8 \mathrm{~mm}$ ). Prior to the degradation test, the initial (time zero) dry mass was measured after drying the samples. Then they were moved to multi-plates containers and $3 \mathrm{~mL}$ of $\mathrm{NaOH} 0.01$ molar $(\mathrm{pH}=12.3)$ was added to each sample and incubated at $37^{\circ} \mathrm{C}$. The $\mathrm{NaOH}$ was changed every week. At days 2, 4, 7, 14, and 28, four samples per each hydrogel group were taken, dried, and weighed them with the same procedure as for time zero.

\section{Mechanical properties and weight loss of hydrogels under cyclic loading}

Since we developed these HEMA-based hydrogels for loadbearing applications, in order to study the degradation in this situation, we evaluated the mechanical properties and weight loss of hydrogels under cyclic loading. We considered two groups of hydrogels. In the first group, a 1000 cyclic compression load was applied every week while no load was applied in the second group. The cyclic compression consisted of a $15 \%$ deformation amplitude at $1 \mathrm{~Hz}$. We used a multi-piston set up, previously designed in our group $^{31}$ to be able to apply load simultaneously on multiple samples. In both groups, hydrogels were incubated at $37^{\circ} \mathrm{C}$ and immersed in $3 \mathrm{~mL}$ PBS ( $\mathrm{pH}=7.4)$. We changed the PBS every 2 weeks. We monitored the elastic modulus (six samples for each of the four hydrogel groups) and weight loss (four samples for each of the four hydrogel groups) every month during nine months following the same procedures as described under the sections 2.4.

\section{Molecular weight of degradation products}

We extracted the degradation products from the PBS media in which the samples were incubated during the degradation study made under the mechanical loading (section 2.6). PBS was collected from each of the four hydrogel groups every month after 4 months of starting the degradation study and was freeze-dried. After freeze-drying, we obtained the degradation products as white powders. The powders were analyzed with Gel Permeation Chromatography (GPC) facility (GPC 50 Agilent, Santa Clara, CA, USA) to quantify the molecular weight of the degradation products. ${ }^{32,33}$ The Eluent for GPC consisted of Milli-Q water and 10\% MeOH. A conventional calibration was performed with RI (Nicolet Magna-ir 
560, Ontario, Canada) and viscometer (Alpha L, Barcelona, Spain). The standards range was 1010 to 278100 Da.

\section{Structure of hydrogels obtained by SEM}

The structure of the hydrogels was obtained using a Scanning Electron Microscope (SEM). Cross-sections of the hydrogels were obtained by cracking frozen gels. We prepared a set of swollen new samples of S1, S2, S3, and S4 and a set of samples partially degraded after 6 months under weekly cyclic loading. Samples were dried by freezedrying, stuck on SEM pads and cross-section of the midregion of the sample were imaged using an electron microscope (Zeiss Merlin, Oberkochen, Germany) at an accelerating voltage of $0.8 \mathrm{kV}$ and $2.5 \mathrm{Kx}$ magnification, with an aperture of $5 \mathrm{~mm}$ and working distance of $6 \mathrm{~mm}$.

Biocompatibility of hydrogels and degradation products The biocompatibility of the developed hydrogels was evaluated by a direct contact test. Cylindrical hydrogels (three samples for each of the four hydrogel groups) were placed separately in the middle of a $60 \mathrm{~mm}$ diameter petri dish and primary human chondrocyte cells isolated and characterized in our group ${ }^{34}$ were seeded around the samples $\left(3000\right.$ cell $\left./ \mathrm{cm}^{2}\right)$. Totally, $5 \mathrm{~mL}$ of cell culture medium was added to each petri dish. After 1 week, cells were fixed by adding $1 \mathrm{~mL}$ methanol to each plate for $30 \mathrm{~s}$ following by $1 \mathrm{~mL}$ diluted Giemsa solution to color the cells. Microscopy of cells on the surface of the hydrogels and on petri dish was performed in order to visualize fixed, colored cells (ZEISS Axiovert 100, Germany).

In a second test, we evaluated the cytotoxicity of the degradation products. The degradation products were collected at different time points from HEMA-DMHA hydrogels (S1 and S3) or HEMA-DMHA-Tetrakis hydrogels (S2 and S4) obtained in section 2.7. Different concentrations of degradation products $(0 \mathrm{mg} / \mathrm{ml}, 0.1 \mathrm{mg} / \mathrm{ml}, 0.5 \mathrm{mg} / \mathrm{ml}, 3 \mathrm{mg} / \mathrm{ml})$ were added in the cell culture medium. The cells' proliferation exposed to the different concentrations was evaluated using a CellTiter assay following the standard manufacturer protocol (CellTiter 96® Aqueous, Promega, Fitchburg, WI) and an absorbance reading at $490 \mathrm{~nm}$ with a spectrophotometer (Wallac Victor2, 1420, Turku, Finland). The CellTiter assay was performed every day for 3 days.

\section{Statistical test}

We used an ANOVA test to determine if a significant difference ( $p$ values $<0.05$ ) is present between each of the four hydrogel groups regarding different parameters like crosslinker, water ratio, presence of Tetrakis, and weekly mechanical load. In conjunction with ANOVA we did a Tukey-Kramer post-hoc test to find means that are significantly different from each other.

\section{RESULTS}

\section{Swelling and mechanical evaluation of hydrogels}

The equilibrium and initial water contents of the hydrogels are reported in Table II. There was a significant difference between the equilibrium and initial water contents in each
TABLE II. Initial and Equilibrium Water Contents

\begin{tabular}{lcc}
\hline $\begin{array}{l}\text { Sample } \\
\text { name }\end{array}$ & $\begin{array}{c}\text { Equilibrium } \\
\text { Water Content (\%) }\end{array}$ & $\begin{array}{c}\text { Initial Water } \\
\text { Content (\%) }\end{array}$ \\
\hline S1 & $51.51 \pm 1.97$ & $41.39 \pm 2.03$ \\
S2 & $59.66 \pm 8.99$ & $39.57 \pm 1.32$ \\
S3 & $133.20 \pm 7.32$ & $63.07 \pm 4.34$ \\
S4 & $149.36 \pm 11.37$ & $59.25 \pm 5.45$ \\
\hline
\end{tabular}

Significant differences were observed between the equilibrium and the initial water contents in each group $(p<0.001)$. The addition of Tetrakis, a decrease in cross-linker ratio or an increase in water ratio significantly increased the equilibrium water content $(p<0.04)$.

group of hydrogels $(p<0.001)$. However, the hydrogels with $1 \%$ cross-linker and $65 \%$ water ratios (S3 and S4) had higher water content at the equilibrium state compared to the hydrogels with $10 \%$ cross-linker and $40 \%$ water (S1 and S2) $(p<0.001)$. This confirms that decreasing the amount of cross-linker and increasing the water ratio increase the swelling of the hydrogels. The hydrogels containing Tetrakis (S2 and S4) had slightly higher equilibrium water content compared to the hydrogels without Tetrakis (S1 and S3) $(p<0.04)$. However, the effect of the crosslinker and the water ratios on the swelling of the hydrogels was more significant than the effect of Tetrakis $(p<0.001)$.

Mechanical characterization of the hydrogels highlights that the elastic modulus highly depends on cross-linker and water ratios. Figure 2(A) shows that the elastic modulus was 10 times lower in hydrogels with less cross-linker and higher water ratios (S1: $2.5 \pm 0.21 \mathrm{MPa}$ vs. S3: $0.23 \pm 0.019$ MPa $(p<0.001)$. The addition of Tetrakis decreased the elastic modulus of the hydrogels to more than half of its original value (S2: $0.85 \pm 0.026 \mathrm{MPa}$ vs $\mathrm{S} 1: 2.5 \pm 0.21 \mathrm{MPa}$ and S4: $0.094 \pm 0.009 \mathrm{MPa}$ vs S3: $0.23 \pm 0.019 \mathrm{MPa}$ ) $(p<0.001)$. However, all hydrogels showed high viscous properties [damping ratio greater than 0.6, Figure 2(B)]. While the addition of Tetrakis slightly increased the damping ratio, there was no significant difference between the damping ratios of the different groups $(p>0.3)$.

\section{Accelerated degradation study}

Figure 3 shows the weight loss by the hydrogel films incubated in $\mathrm{NaOH} 0.01$ molar $(\mathrm{pH}=12.3)$ over four weeks. The addition of Tetrakis significantly increased the amount of weight loss at each time point $(p<0.001)$. Also, the weight loss by hydrogels with $1 \%$ cross-linker and $65 \%$ water ratios (S3 and S4) was higher than the other two groups (S1 and S2) $(p<0.001)$. However, the effect of adding Tetrakis was more significant than the effect of cross-linker and water ratios on the weight loss $(\mathrm{p}<0.001)$. Hydrogels containing Tetrakis, $1 \%$ cross-linker and $65 \%$ water (S4) completely degraded in one week.

\section{Mechanical properties and weight loss by hydrogels under cyclic loading}

Figure 4 shows the changes in the elastic modulus of all hydrogel groups (S1, S2, S3, and S4) over nine months degradation in PBS, with or without weekly cyclic mechanical 

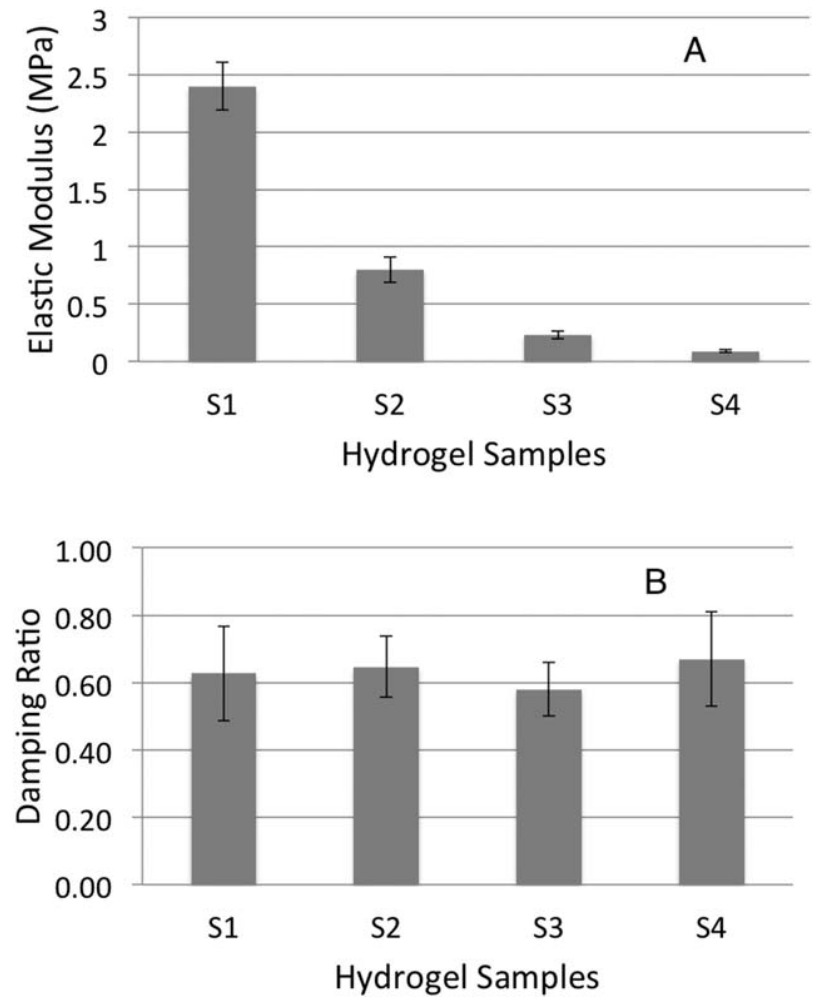

FIGURE 2. A: The elastic modulus of the four groups of hydrogels. Hydrogels with $1 \%$ cross-linker ratio and $65 \%$ water content (S3 and S4) had smaller elastic modulus than the ones with $10 \%$ cross-linker ratio and $40 \%$ water content (S1 and S2) $(p<0.001)$. Hydrogels containing Tetrakis (S2 and S4) had lower elastic modulus compared to those without Tetrakis (S1 and S3) $(p<0.001)$. B: The damping ratios of the four groups of hydrogels. There was no significant difference between the damping ratios of the different groups $(p>0.3)$.

loading. The elastic modulus decreased over time, in some cases, by [mt]90\%. For each group, applying a mechanical load induced a significant decrease in the elastic modulus value at each time point $(p<0.001)$. However, changing the cross-linker and water ratios and adding Tetrakis had no significant influence on the final percentage of elastic modulus loss after nine months $(p>0.1)$. Furthermore, we observed that the rate of loss of elastic modulus (the slope of the curve) was higher during the first two months of degradation for all hydrogels.

In Figure 5, the weight loss by all hydrogel groups during the nine months of degradation, with or without weekly cyclic loading, is reported. For hydrogels S1, S2, and S4, applying mechanical load resulted to a significant increase in the amount of weight loss at each time point $(p<0.001)$. However for the group S3, this effect was observed only after five months. The addition of Tetrakis significantly increased the amount of weight loss in all hydrogel groups over the entire degradation period $(p<0.001)$. A correlation was found between applying mechanical loading and adding Tetrakis $(p<0.01)$, suggesting that having both parameters highly increases the degradation. However, from our results, the effect of cross-linker and water ratios on the amount of weight loss was not clear. While for hydrogels containing Tetrakis the amount of weight loss after nine months was greater for hydrogels with $1 \%$ cross-linker ratio and $65 \%$ water content (S4) compared to hydrogels with $10 \%$ crosslinker ratio and $40 \%$ water content (S2), we observed an opposite phenomenon in hydrogels without Tetrakis (S3 and S1). Furthermore, we observed that for hydrogels containing Tetrakis the slope of the weight loss curves increased in the later months of degradation, while for the hydrogels without Tetrakis the slope of the curves decreased. This suggests that the degradation of hydrogels containing Tetrakis increases in the later months, while this is less likely to happen in hydrogels without Tetrakis.

\section{Molecular weight of degradation products}

The results of GPC test showed that there was no significant difference in the molecular weight of degradation products between the hydrogels with $10 \%$ cross-linker (S1 and S2) and those with $1 \%$ cross-linker ratio (S3 and S4) ( $p>0.5)$. Therefore, we considered highly cross-linked and little cross-linked groups together and we only separated groups by whether or not they contained Tetrakis. All the samples without Tetrakis had a peak in the GPC graph ranging between 7000 and $9000 \mathrm{kDa}(7668 \pm 816 \mathrm{kDa})$. However two samples had also a second peak, one at $4683 \mathrm{kDa}$, and the other at $2966 \mathrm{kDa}$. For samples with Tetrakis we detected two peaks for all samples, one peak between 4500 and $7500 \mathrm{kDa}(6096 \pm 1544 \mathrm{kDa})$, and another peak between 2500 and $3200 \mathrm{kDa}(2820 \pm 351 \mathrm{kDa})$.
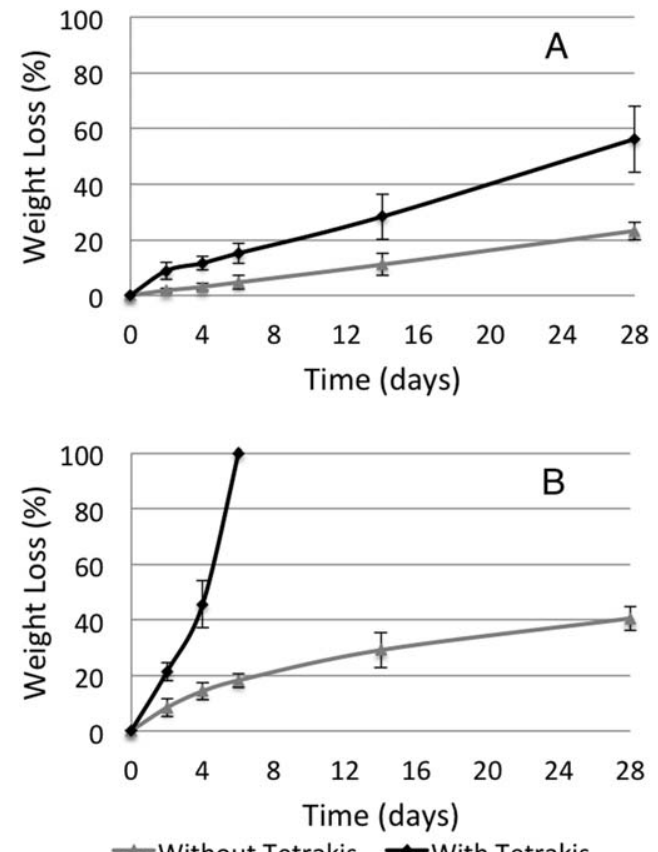

FIGURE 3. The amount of weight loss of the hydrogels in an accelerated degradation study. A: Hydrogels with $10 \%$ cross-linker ratio and $40 \%$ water content (S1 and S2), B: hydrogels with $1 \%$ cross-linker ratio and $65 \%$ water content (S3 and S4). The addition of Tetrakis significantly increased the amount of weight loss at each time point $(p<0.001)$. Hydrogels with a lower cross-linker ratio and a higher water content (S3 and S4) lost weight faster than the other groups (S1 and S2) $(p<0.001)$. Hydrogels in group S4 completely degraded after 1 week. 

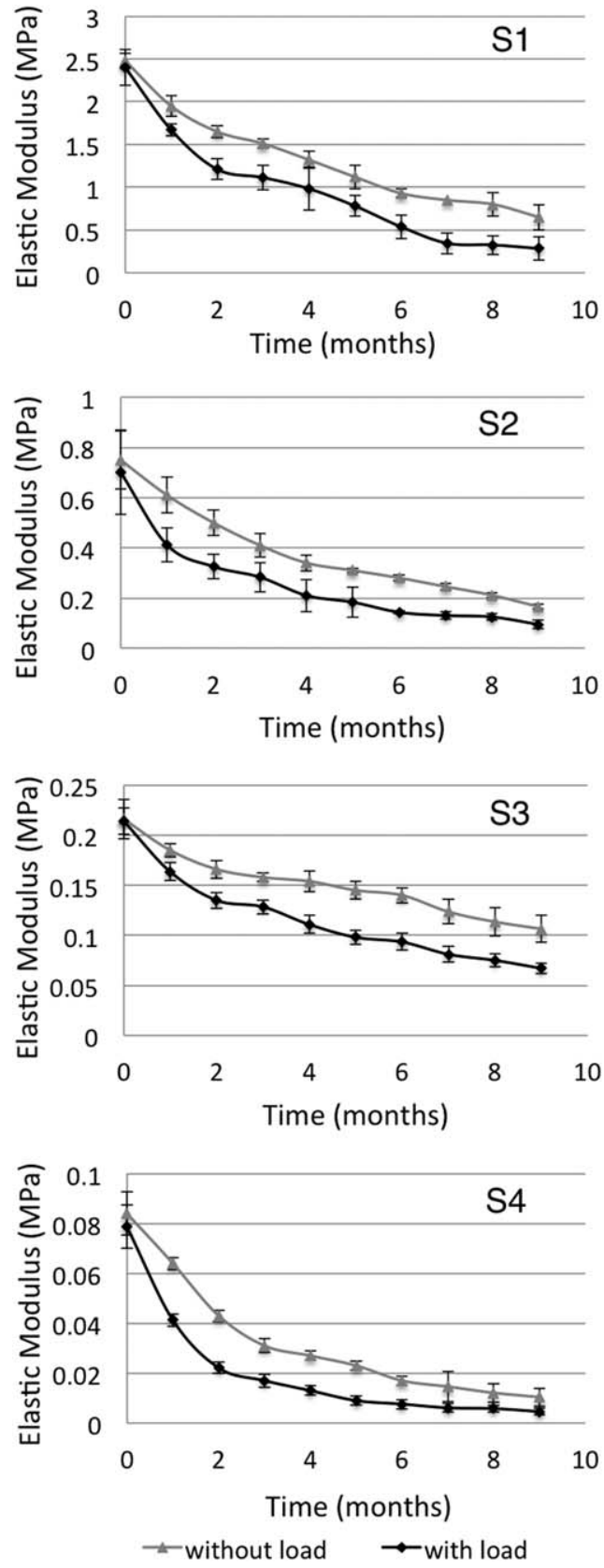

FIGURE 4. Evolution of the elastic modulus of the hydrogels (S1, S2 S3, and S4) during degradation in PBS, with and without applying weekly cyclic loading. For each group, applying a mechanical load induced a decrease in the elastic modulus value at each time point $(p<0.001)$. Changing the cross-linker and water ratios and adding Tetrakis had no significant influence on the final percentage of elastic modulus loss after nine months $(p>0.1)$. The final percentage of elastic modulus loss after nine months was S1: $88.23 \pm 7.54 \%$, S2: $86.57 \pm 5.35 \%$, S3: $78.96 \pm 8.32 \%$, and S $4: 94.15 \pm 8.76 \%$.

\section{Structure of hydrogels obtained by SEM}

The morphology and porosity of the hydrogels were examined by SEM (Figure 6). We could not observe the porous structure of highly cross-linked hydrogels (S1 and S2) since the structure was too dense to be observed with SEM at the voltage of $0.8 \mathrm{kV}$ and increasing the voltage ruined the samples. In contrast, we were able to see the porous structure of hydrogels with $1 \%$ cross-linker and $65 \%$ water containing or not Tetrakis (S3 and S4). As Figure 6 shows, in the presence of Tetrakis, the hydrogel has larger and rounder pores, which seem to be less interconnected to each other [Figure 6(A,B)]. We also examined these hydrogels after six months of degradation under weekly cyclic load. The
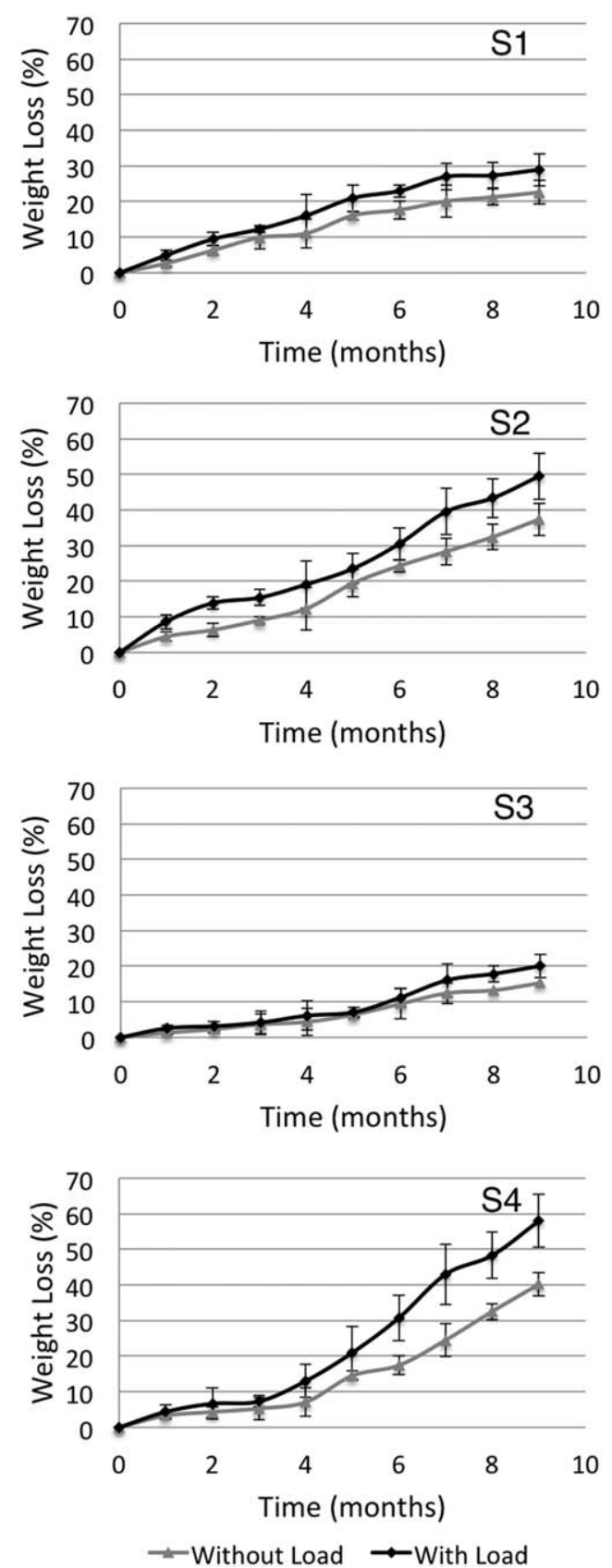

FIGURE 5. The amount of weight loss of the hydrogels (S1, S2, S3, and S4) during degradation in PBS, with and without applying weekly cyclic loading. For each group, applying a mechanical load increased the amount of weight loss at each time point $(p<0.001)$ except the first 5 months of the group S3. Also, the groups with Tetrakis (S2 and S4) showed a greater weight loss compared to the groups without Tetrakis (S1 and S3) $(p<0.001)$. Changing the cross-linker and water ratios had no significant influence on the amount of weight loss $(p>0.5)$. 


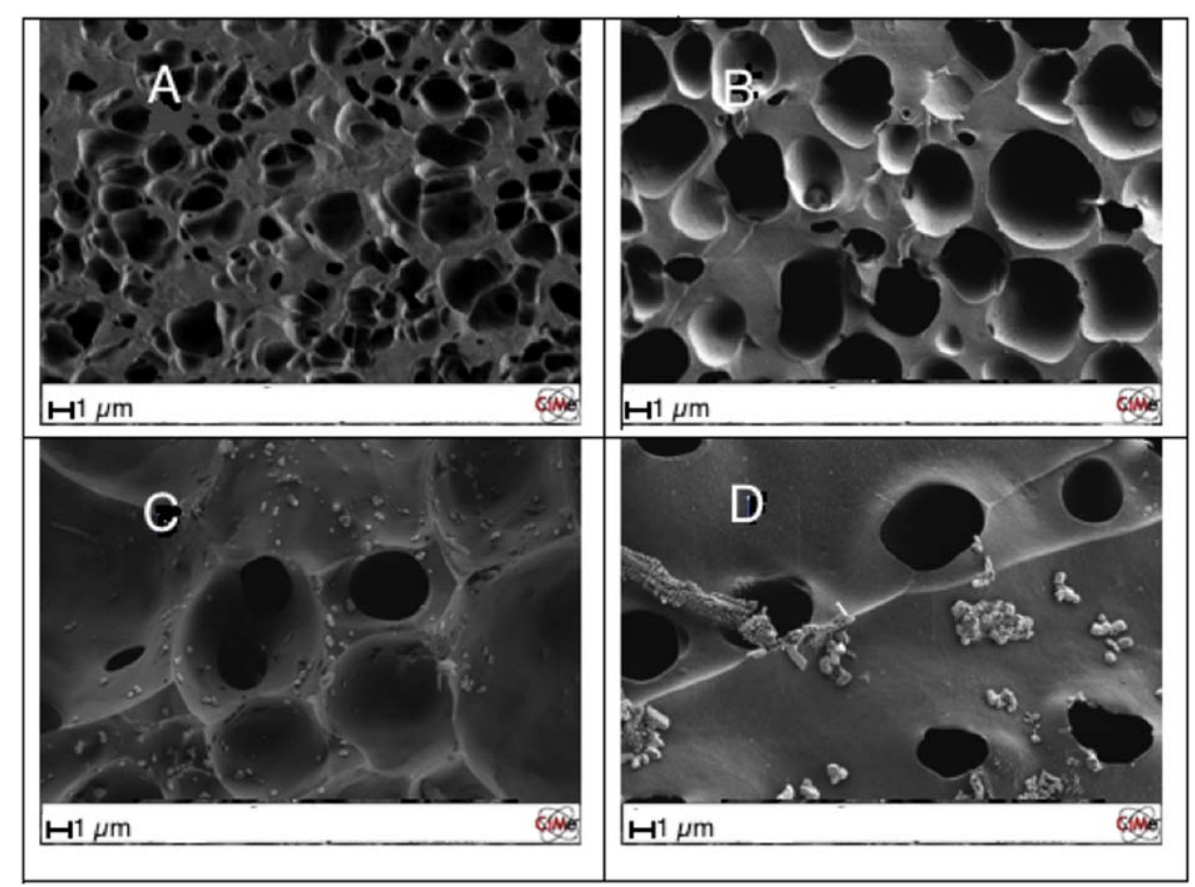

FIGURE 6. Scanning Electron Microscopy pictures of the hydrogels. A: S3 at zero time point. B: S4 at zero time point. C: S3 after six months of degradation under cyclic loading. D: S4 after six months of degradation under cyclic loading.

structure of the partly degraded hydrogels was highly porous and interestingly we could visually detect the particles of the degradation product on the wall of the pores [Figure 6(C,D)]

Biocompatibility of hydrogels and degradation products The morphology and proliferation of the cells in contact with the hydrogel samples were similar to those seeded on standard cell culture plates. Figure 7 shows a typical Giemsa staining for hydrogel S2. The microscopic picture of the colored cells demonstrated that their density and shape in contact with the hydrogel (Zone 1) were similar to the areas far from the hydrogel (Zone 2) on the cell culture plate.
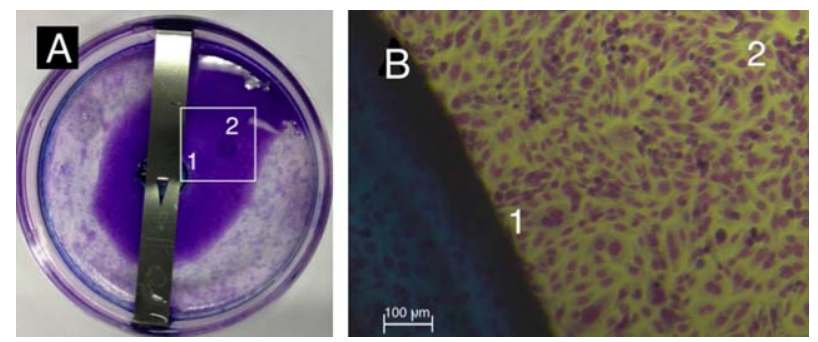

FIGURE 7. Giemsa staining of the sample $\mathrm{S} 2$ to visualize cells' distribution around the hydrogel. A: an overview of the area of interest (white rectangle). B: microscopic picture of the area of interest. In each image, the label (1) corresponds to the cells near the hydrogel and the label (2) corresponds to the cells on cell culture plate far from the hydrogel.
The evaluation of the cytotoxicity of the degradation products over three days confirmed that these products did not affect the cells proliferation. In Figure 8, we can observe that, at each day, there was no significant difference between the absorbance signal of cells cultured in mediums containing different concentrations of degradation products and the control group, which contains no degradation product $(p>0.25)$.

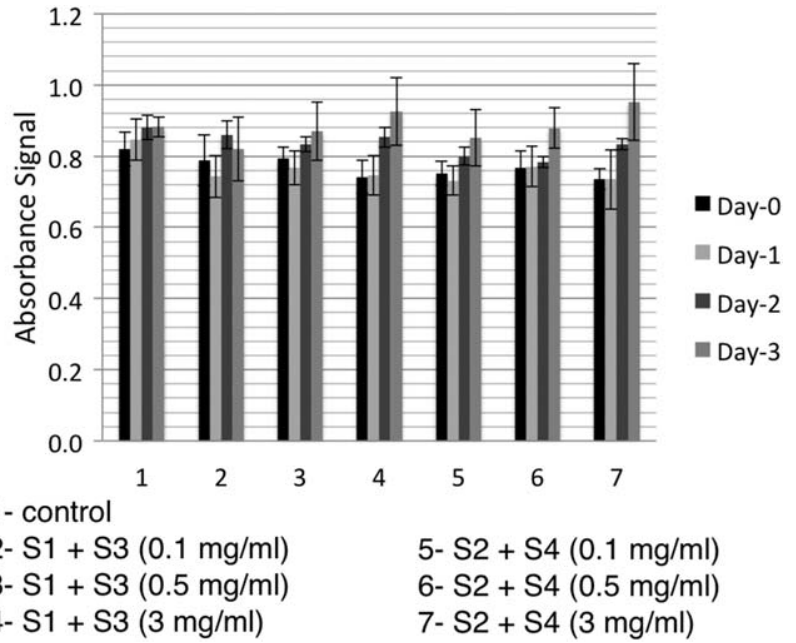

FIGURE 8. Spectrophotometry absorbance signal for CellTiter test, when different concentrations of degradation products from HEMADMHA hydrogels (S1 and S3) or HEMA-DMHA-Tetrakis hydrogels (S2 and S4) were exposed to cells for 3 days. No significant difference could be found between the mean values of the absorbance signal of each group containing the degradation products and the mean values of the control groups at each day $(p>0.25)$. 


\section{DISCUSSION}

We developed a biodegradable photo-crosslinked hydrogel based on HEMA with high mechanical properties for loadbearing applications. Our original formulation consisted of HEMA cross-linked with a biodegradable N, O-dimethacryloyl hydroxylamine (DMHA) molecule and a tetra-functional chain transfer agent pentaerythritol tetrakis in the backbone of the hydrogel. We studied the degradation under cyclic mechanical loading, which provides a more realistic environment to evaluate the degradation of hydrogels in load-bearing conditions. Mechanical loading can increase water circulation inside the porous structure of hydrogels, which favors hydrolysis and helps to remove the degradation products.

The obtained HEMA-DMHA-Tetrakis hydrogels degraded in low molecular weight products over a prolonged time period. With respect to its mechanical properties, the elastic modulus of the hydrogels decreased at the initial stage of the degradation process. Since the elastic modulus of the hydrogels is proportional to their cross-linker ratio, ${ }^{35}$ we concluded that the decrease in the elastic modulus during the early months of degradation was related to the hydrolysis of the DMHA cross-linker. The increase of the weight loss rate after several months for the hydrogels containing Tetrakis confirmed that in these hydrogels, their backbone started to break apart during a second stage of degradation. At this stage, the hydrogels began to lose their mechanical integrity. If the hydrogels did not contain any Tetrakis, the degradation could not be completed as in this situation, after the hydrolysis of the cross-linker, the structure of the hydrogels consisted of long nondegradable PHEMA chains which could coil and keep their integrity via some hydrophobic interactions. ${ }^{36}$ The presence of Tetrakis allowed the long PHEMA chains to be broken into shorter-length molecules presenting a star structure. This effect has already been described by Chiellini et al. ${ }^{25}$ Having low molecular weight degradation products is one of the most important aspects when developing biodegradable hydrogels. Since the degradation products of our hydrogels were $<10 \mathrm{kDa}$, they could be cleared out of body. 6,27

It should be noticed however that we did not observe a complete degradation of the hydrogels over the nine months degradation study. Slow degradation rate of our hydrogels and their high mechanical properties could anyway be desirable for scaffolding in load-bearing applications. Longterm degradation mirrors the rate of tissue development in load-bearing tissues like cartilage and nucleus pulposus where the healing process is slow. ${ }^{37-39}$ In parallel, the high damping properties observed in the develop hydrogels provided load and crack resistance under the loading conditions of such tissues. ${ }^{12,18}$

It has been already shown that HEMA-based hydrogels can partially degrade when they are cross-linked to some natural polysaccharides molecules such as chitosan, ${ }^{22}$ dextran-based molecules, ${ }^{7,40}$ hyaluronic acid and poly(lactic acid) PLA. ${ }^{35}$ Due to the complexity of the functionalization of most of these molecules, their high molecular weight, and their mechanism of cross-linking, resulting hydrogels usually have very poor mechanical properties and cannot be used for load-bearing applications. Furthermore, in most cases, the high molecular weight of the resulting degradation products has been reported to be an issue. For example, Atzet et al. has developed a biodegradable PHEMA gels cross-linked with polycaprolactone (PCL) which presented high and tunable mechanical properties, but also had degradation products with high molecular weight. ${ }^{6}$ The degradation properties of DMHA, which we used, have been already characterized in the literature. ${ }^{28,30,41}$ We chose this molecule because it is a hydrolysable short-length molecule similar in size to HEMA monomers. We have previously shown that using cross-linkers similar in size to the HEMA monomers increased the viscous properties of the HEMA-based hydrogels, which favored then their crack resistance. ${ }^{18}$ DMHA undergoes base catalyzed hydrolysis at $\mathrm{pH}$ values above 5 , thus it is a useful cross-linker which can be cleaved at a physiological $\mathrm{pH}$ of $7.4 .^{28,30}$ Huang et al. reported partial weight and stiffness loss in hydrogels made of PHEMA cross-linked by DMHA. ${ }^{26}$ Indeed, these authors showed that the weight loss reached a constant value $(20 \%)$ after several months. Our results presented the same degradation profile for HEMA-DMHA hydrogels containing no Tetrakis. As mentioned before, this is due to the long interconnected PHEMA chains in the backbone of hydrogels. Based on the results of this study, using Tetrakis in the backbone of hydrogels solved this issue. Chiellini et al. has already used Tetrakis molecules to increase the degradation properties of PHEMA hydrogels. ${ }^{25}$ However, in their study, they used poly(ethylene glycol di-methacrylate) (PEGDM) as a crosslinker, which is nondegradable. To the best of our knowledge, no other study has tried to use the advantage of Tetrakis molecules in PHEMA hydrogels.

There are some limitations in our study. We evaluated the degradation properties of two HEMA-DMHA-Tetrakis hydrogels as a model system. The first hydrogel was obtained with a low cross-linker ratio and high water content, which can mimic soft-tissues like nucleus pulposus. The second one was made with a high cross-linker ratio and low water content, with resulting mechanical properties similar to articular cartilage. We did not study the effects of these two parameters (cross-linker ratio and water content) separately on the degradation and the mechanical properties of the hydrogels. The effects of these two parameters on the mechanical properties of HEMA-based hydrogels have already been studied for similar geometry cross-linkers like ethylene glycol dimethacrylate (EGDMA). ${ }^{3,16-18}$ Another limitation is related to the GPC method used to measure the molecular weight of the degradation products. This method can only identify the different molecular weights of material composing the hydrogels, but it cannot bring any information on their respective distribution. Nevertheless, the presence of peaks at lower molecular weights in the GPC signal of hydrogels containing Tetrakis strongly suggests that using this molecule decreases the molecular weight of the degradation products. Furthermore, we were still able to show that the molecular weight of all degradation products even from hydrogels without Tetrakis was smaller than the required limit for being cleaned out of the body. 


\section{CONCLUSIONS}

In conclusion, the new formulation of the HEMA-DMHATetrakis hydrogels allows us to obtain a promising slow degrading biomaterial presenting low molecular weight degradation products, along with tunable mechanical properties. With such properties, the obtained hydrogels could overcome the existing limitations in the application of HEMA-based hydrogels in tissue engineering and drug delivery, especially for load-bearing applications.

\section{REFERENCES}

1. Kamath KR, Park K. Biodegradable hydrogels in drug delivery. Adv Drug Deliv Rev 1993;11:59-84.

2. Drury JL, Mooney DJ. Hydrogels for tissue engineering: scaffold design variables and applications. Biomaterials 2003;24:4337-4351.

3. Peppas NA, Hilt JZ, Khademhosseini A, Langer R. Hydrogels in biology and medicine: From molecular principles to bionanotechnology. Adv Mater 2006;18:1345-1360.

4. Oliveira JT, Gardel LS, Rada T, Martins L, Gomes ME, Reis RL. Injectable gellan gum hydrogels with autologous cells for the treatment of rabbit articular cartilage defects. Journal of Orthopaedic Research 2010;28:1193-1199.

5. Moffat KL, Marra KG. Biodegradable poly(ethylene glycol) hydrogels crosslinked with genipin for tissue engineering applications. J Biomed Mater Res Part B: Appl Biomater 2004;71:181-187.

6. Atzet S, Curtin S, Trinh P, Bryant S, Ratner B. Degradable poly (2hydroxyethyl methacrylate)-co-polycaprolactone hydrogels for tissue engineering scaffolds. Biomacromolecules 2008;9:3370-3377.

7. van Dijk-Wolthuls WNE, Tsang SKY, Kettenes-van den Bosch W.E. Hennink JJ. A new class of polymerizable dextrans with hydrolyzable groups: Hydroxyethyl methacrylated dextran with and without oligolactate spacer. Polymer 1997;38:6235-6242.

8. Gunatillake PA, Adhikari R. Biodegradable synthetic polymers for tissue engineering. Eur Cell Mater 2003;5:1-16.

9. De Jong S, Van Eerdenbrugh B, van Nostrum Cv, Kettenes-Van Den Bosch J, Hennink W. Physically crosslinked dextran hydrogels by stereocomplex formation of lactic acid oligomers: Degradation and protein release behavior. J Control Release 2001;71:261-275.

10. Bae KH, Wang L-S, Kurisawa M. Injectable biodegradable hydrogels: progress and challenges. Journal of Materials Chemistry B 2013;1:5371-5388.

11. Hennink W, Van Nostrum C. Novel crosslinking methods to design hydrogels. Adv Drug Deliv Rev 2012;64:223-236.

12. Sun JY, Zhao X, Illeperuma WR, Chaudhuri O, Oh $\mathrm{KH}$, Mooney DJ, et al. Highly stretchable and tough hydrogels. Nature 2012; 489:133-136.

13. Naficy S, Brown HR, Razal JM, Spinks GM, Whitten PG. Progress toward robust polymer hydrogels. Australian J Chem 2011;64: 1007-1025

14. Anseth KS, Bowman CN, Brannon-Peppas L. Mechanical properties of hydrogels and their experimental determination. Biomaterials 1996;17:1647-1657.

15. Homsy CA. Bio-Compatibility in selection of materials for implantation. J Biomed Mater Res 1970;4:341-356.

16. Peppas NA, Moynihan HJ, Lucht LM. The structure of highly crosslinked poly(2-hydroxyethyl methacrylate) hydrogels. J Biomed Mater Res 1985;19:397-411.

17. Baker MV, Brown DH, Casadio YS, Chirila TV. The preparation of poly(2-hydroxyethyl methacrylate) and poly\{(2-hydroxyethy methacrylate)-co-[poly(ethylene glycol) methyl ether methacrylate]\} by photoinitiated polymerisation-induced phase separation in water. Polymer 2009:50:5918-5927.

18. Nassajian Moghadam M, Pioletti DP. Improving hydrogels' toughness by increasing the dissipative properties of their network. J Mech Behav Biomed Mat: 2015;41:161-167.

19. Bayramoğlu G, Arıca MY. A novel pH sensitive porous membrane carrier for various biomedical applications based on $\mathrm{pHEMA} / \mathrm{chi}$ tosan: Preparation and its drug release characteristics. Macromol Symp 2003;203:213-218.

20. Casadio YS, Brown DH, Chirila TV, Kraatz H-B, Baker MV. Biodegradation of Poly(2-hydroxyethyl methacrylate) (PHEMA) and
Poly\{(2-hydroxyethyl methacrylate)-co-[poly(ethylene glycol) methyl ether methacrylate]\} Hydrogels Containing Peptide-Based Cross-Linking Agents. Biomacromolecules 2010;11:2949-2959.

21. Cadee JA, De Kerf M, De Groot CJ, Den Otter W, Hennink WE. Synthesis, characterization of 2-(methacryloyloxy)ethyl-(di-) Ilactate and their application in dextran-based hydrogels. Polymer 1999;40:6877-6881.

22. Verestiuc L, Nastasescu O, Barbu E, Sarvaiya I, Green KL, Tsibouklis J. Functionalized chitosan/NIPAM (HEMA) hybrid polymer networks as inserts for ocular drug delivery: Synthesis, in vitro assessment, and in vivo evaluation. J Biomed Mater Res Part A 2006;77:726-735.

23. Han $Y$, Lee $E$, Ji B. Mechanical properties of semi-interpenetrating polymer network hydrogels based on poly(2-hydroxyethyl methacrylate) copolymer and chitosan. Fibers Polym 2008;9:393-399.

24. Ng L-T, Swami S. IPNs based on chitosan with NVP and NVP/ HEMA synthesised through photoinitiator-free photopolymerisation technique for biomedical applications. Carbohyd Polym 2005; 60:523-528.

25. Chiellini F, Petrucci F, Ranucci E, Solaro R. Bioerodible hydrogels based on 2-hydroxyethyl methacrylate: Synthesis and characterization. J Appl Polym Sci 2002;85:2729-2741.

26. Huang J, Ten E, Liu G, Finzen M, Yu W, Lee JS, et al. Biocomposites of pHEMA with HA-TCP $(60 / 40)$ for bone tissue engineering: Swelling, hydrolytic degradation, and in vitro behavior. Polymer 2013;54:1197-1207.

27. Jiang $X$, Lok MC, Hennink WE. Degradable-brushed pHEMApDMAEMA synthesized via ATRP and click chemistry for gene delivery. Bioconjugate Chem 2007;18:2077-2084.

28. South $A B$, Lyon LA. Direct observation of microgel erosion via inliquid atomic force microscopy. Chem Mater 2010;22:3300-3306.

29. [29] Vogel A, Pioletti DP. Thermomechanical hysteresis of biological and synthetic hydrogels: Theory, characterisation, and development of a novel deformation calorimeter: PhD thesis, ecole polytechnique federale de Lausanne; 2011.

30. Ulbrich K, Subr V, Seymour LW, Duncan R. Novel biodegradable hydrogels prepared using the divinylic crosslinking agent N,Odimethacryloylhydroxylamine. I. Synthesis and characterisation of rates of gel degradation, and rate of release of model drugs, in vitro and in vivo. J Control Release 1993;24:181-190.

31. Abdel-Sayed P, Darwiche SE, Kettenberger U, Pioletti DP. The role of energy dissipation of polymeric scaffolds in the mechanobiological modulation of chondrogenic expression. Biomaterials 2014;35:1890-1897.

32. Striegel A, Yau WW, Kirkland JJ, Bly DD. Modern Size-Exclusion Liquid Chromatography: Practice of Gel Permeation and Gel Filtration Chromatography. Wiley 2009;2:145-180.

33. Gellerstedt G. Gel Permeation Chromatography. Methods in Lignin Chemistry. Springer; 1992;21:487-497.

34. Darwiche S, Scaletta C, Raffoul W, Pioletti DP, Applegate LA. Epiphyseal chondroprogenitors provide a stable cell source for cartilage cell therapy. Cell Med 2012;4:23-32.

35. Hennink WE, van Nostrum CF. Novel crosslinking methods to design hydrogels. Adv Drug Deliv Rev 2012;64:223-236.

36. Refojo MF. Hydrophobic interaction in poly(2-hydroxyethyl methacrylate) homogeneous hydrogel. J Polym Sci Part A-1: Polym Chem 1967;5:3103-3113.

37. A. Getgood, R. Brooks, L. Fortier, Rushton N. Articular cartilage tissue engineering, TODAY'S RESEARCH, TOMORROW'S PRACTICE? J Bone Joint Surg B 2009;91:565-576.

38. Chung C, Burdick JA. Engineering cartilage tissue. Adv Drug Deliv Rev 2008;60:243-262.

39. Moghadam MN, Kolesov V, Vogel A, Klok H-A, Pioletti DP. Controlled release from a mechanically-stimulated thermosensitive self-heating composite hydrogel. Biomaterials 2014;35:450-455.

40. Van Tomme SR, van Nostrum CF, de Smedt SC, Hennink WE. Degradation behavior of dextran hydrogels composed of positively and negatively charged microspheres. Biomaterials 2006;27: 4141-4148.

41. Smith $M H$, South $A B$, Gaulding JC, Lyon LA. Monitoring the erosion of hydrolytically-degradable nanogels via multiangle light scattering coupled to asymmetrical flow field-flow fractionation. Analytical Chemistry 2009;82:523-530. 